\title{
Statistical Magnitude Analysis and Distance Determination of the Nearby F8V Stars
}

\author{
Hany R. Dwidar
Astronomy, Meteorology and Space Science Dept. \\ Hany R. Dwidar
Astronomy, Meteorology and Space Science Dept. \\ Faculty of Science, Cairo University \\ Giza, Egypt \\ hanyryd@gmail.com
}

\begin{abstract}
The present paper is of three folds. First, to provide some basic descriptive statistics parameters for the apparent and absolute magnitudes of the nearby stars of spectral type F8V stars. Second, to establish the frequency functions $\Phi(M)$ and $\Psi(m)$ of the absolute and apparent magnitudes for these stars. Third, to compute the distance $r$ of these stars as a system assuming that they scatter around a mean absolute magnitude in a Gaussian distribution. The accuracy of the numerical results is satisfactory thus, the percentage error between $r$ and the mean value is less than $0.7 \%$.
\end{abstract}

Keywords-distance determinations; spectral types; frequency functions; statistical magnitude analysis

\section{INTRODUCTION}

An F-type main sequence star (F V), commonly called a yellow-white dwarf, is a main sequence (hydrogen-burning) star of spectral type $\mathrm{F}$ and luminosity class V. These stars have from 1.0 to 1.4 times the mass of the Sun and surface temperatures between 6,000 and 7,600 K [1]. A star of spectral type $\mathrm{F}$ has a spectrum that shows strong absorption lines of ionized calcium, more prominent than the hydrogen lines. Moderately strong lines due to iron and other heavier elements are also evident. The lines of neutral atoms for the stars of spectral type F are weak compared to those of ionized atoms. The hydrogen lines are stronger, attaining their maximum intensities in A-type stars. Thereafter, these absorption lines gradually fade as the hydrogen becomes ionized [2].

Main sequence $\mathrm{F}$ stars, of which Procyon is an example, have a surface temperature of 5,800 to $6,900^{\circ} \mathrm{C}$, a mass of 1.2 to 1.6 solar masses, and a luminosity of 2 to 6 times that of the Sun. Relatively nearby, late-type members of this category are generally included in the list of target stars for extra solar planet searches and targeted NASA SETI programs. F-type super giants, of which Canopus and Polaris (the Pole Star) are examples, have a mass up to 12 solar masses and luminosity up to 32,000 times that of the Sun.

The importance of the distance of F8V stars derives from that if its moving stellar vertex and its distance of each member are known, then one can easily determine the velocity of the cluster, the position of its centre [3, 4] and also the distribution of the cluster's members about this centre $[5,6]$.

\author{
Adel Sharaf \\ Department of Astronomy \\ Faculty of Science, King Abdul Aziz University \\ Jeddah, Saudi Arabia \\ sharaf adel@hotmail.com
}

Modern observational astronomy has been characterized by an enormous growth in acquisition stimulated by the advent of new technologies in telescopes, detectors and computations. This new astronomical data give rise to innumerable statistical problems [7].

In the present paper we consider F8V stars and perform the following:

- providing some basic descriptive statistics parameters for the apparent and absolute magnitudes of these stars

- $\quad$ establishing the frequency functions $\Phi(M)$ and $\Psi(m)$ of the absolute and apparent magnitudes for these stars

- computing the distance $r$ of these stars as a system assuming that they scatter around a mean absolute magnitude in a Gaussian distribution.

The accuracy of the numerical results is satisfactory thus, the percentage error between $r$ and the mean value is less than $0.7 \%$.

\section{BASIC MATERIALS}

\section{A. Data}

The source of data is (V/70 A) nearby stars [8] of $(\mathrm{I} / 239)$ Hipparcos and Tycho Catalogues [9, 10]. In Table I of Appendix A, the data for some F8V stars are listed. The columns of the table have the following meaning:

- Column 1: Name with the following acronyms :

o G1 [11].

o GJ [12].

o Wo [13].

- NN newly added stars (number added at CDS)

- Column 2: Vmag (unit mag): Apparent magnitude

- Column 3: B-V(unit mag): Color

- Column 4: U-B(unit mag): Color

- Column 5: plx (unit mas $\equiv$ milli-second of arc): Parallax

- Column 6: $\mathrm{M}_{\mathrm{V}}$ (unit mag): Absolute visual magnitude

B. The distance equation

The following assumptions will be used to derive the distance equation [4]: 
- All the members in a given cosmic group are at the same distance, $r$ parsecs.

- The frequency function for the absolute magnitudes of the members of the cosmic group is

$$
\Phi(M)=\frac{1}{\sigma \sqrt{2 \pi}} e^{-\left(M-M_{0}\right)^{2} / 2 \sigma^{2}}
$$

That is the members scatter around a mean absolute magnitude $M_{0}$ in a Gaussian distribution with dispersion $\sigma$.

- The mean apparent magnitude $\bar{m}$ of the members of the cosmic group and the limiting apparent magnitude $m_{1}$ are related through the quantity $\alpha$ where

$$
\alpha=\frac{m_{1}-\bar{m}}{\sigma}
$$

According to the above assumptions, the distance $r$ and the frequency functions $\Psi(m)$ of the apparent magnitude are given respectively by:

$$
\begin{aligned}
& r=10^{1+\left(m_{1}-M_{0}-\sigma y\right) / 5} \\
& \Psi(m)=\frac{1}{\sigma \sqrt{2 \pi}} e^{-\left(m+5-5 \log r-M_{0}\right)^{2} / 2 \sigma^{2}}
\end{aligned}
$$

where $y$ is the solution of the transcendental equation:

$$
\Lambda(y)=y+e^{-y^{2} / 2}\left\{\sqrt{\frac{\pi}{2}}\left[1+\operatorname{erf}\left(\frac{y}{\sqrt{2}}\right)\right]\right\}^{-1}-\alpha=0,
$$

and, $\operatorname{erf}(z)$ is the error function defined by the integral

$$
\operatorname{erf}(\mathrm{z})=\frac{2}{\sqrt{\pi}} \int_{0}^{z} e^{-t^{2}} \mathrm{~d} t
$$

we call (4) the distance equation.

The relation between $M_{0}$ and $\sigma$ is given by [3]

$$
M_{0}=\bar{M}+1.382 \sigma^{2}
$$

where $\bar{M}$ is the mean absolute magnitudes of the stellar group.

\section{Empirical relation of $\mathrm{M}_{\mathrm{V}}$ versus $(B-V)_{0}$}

An empirical relation between the absolute visual magnitude $M_{\mathrm{v}}$ and $(B-V)_{0}$ is given in [14] in a tabular form (Table II of Appendix A). From this empirical relation one can deduce an interpolating polynomial between $(B-V)_{0}$ and $M_{\mathrm{V}}$ in the form [15]:

$$
(B-V)_{0}=\sum_{j=1}^{20} q_{j} \mathrm{M}_{\mathrm{V}}
$$

where $q$ 's are listed in Table III of Appendix A.

\section{NUMERICAL APPLICATION}

A. Procedure

1. compute the parallax $p$ in second of arc from

$$
p=\mathrm{plx} / 1000
$$

2. compute for each star of the group $(B-V)_{0}$ from its given value of $\mathrm{M}_{\mathrm{V}}$ using (7)

3. compute the absorption $A_{\mathrm{V}}$ for each star of the group from:

$$
A_{\mathrm{v}}=3\left[(B-V)-(B-V)_{0}\right]
$$

4. compute the average $\bar{A}_{\mathrm{V}}$ of $A_{\mathrm{V}}$

5. correct the apparent magnitudes for absorption from

$$
\text { Vmag=Vmag- } \bar{A}_{\mathrm{V}}
$$

6. compute the average value $\overline{\mathrm{M}}_{\mathrm{V}}$ of the absolute magnitudes $\mathrm{M}_{\mathrm{V}}$

7. compute the average value $\bar{r}$ of the individual distances $r_{j}\left(r_{j}=1 / p_{j}\right)$

8. compute the median $r_{\text {median }}$ of the individual distances

9. select start and end values of $\sigma$, say $\sigma_{s}$ and $\sigma_{e}$ respectively. In the present application, $\sigma_{s}=0.5$ and $\sigma_{e}=2$

10. compute the optimum value of $\sigma$ as follows:

a. For $\sigma=\sigma_{s}(0.01) \sigma_{e}$ perform the following calculations:

- $M_{0}=\overline{\mathrm{M}}_{\mathrm{v}}+1.382 \sigma^{2}$

- compute the distance $r$ (in parsec) of the stellar group from the values of Vmag, $M_{0}$ and $\sigma$ as obtained from the statistical method using the algorithm of [4].

- compute the percentage error $f$ in the mean from: $f=|(r-\bar{r}) / \bar{r}| \times 100$

- compute the percentage error $g$ in the median from $g=\left|\left(r-r_{\text {median }}\right) / r_{\text {median }}\right| \times 100$

b. compute the value $\sigma_{1}$ that gives the minimum of the $f^{\prime} s$ values

c. compute the value $\sigma_{2}$ that gives the minimum of the $g^{\prime} s$ values

d. compute the optimum value of $\sigma$ as $\sigma=\left(\sigma_{1}+\sigma_{2}\right) / 2$.

11. compute some basic descriptive statistics parameters for the apparent and absolute magnitudes of the stellar group.

12. compute the measures of the central tendency for the individual distances $r_{j}=1 / p_{j}$ of the stellar group, of these measures are :

- The Mean distance $\bar{r}$ (in parsec) of $r_{j}$

- The Mode of $r_{j}$

- The Median of $r_{j}$

- The Harmonic Mean of $r_{j}$

- The Geometric Mean of $r_{j}$ 
13. From the optimum values of $M_{0}$ and $\sigma$ compute the frequency functions $\Phi(M)$ and $\Psi(m)$ of the absolute and apparent magnitudes [(1) and (3)] of the group.

\section{B. Numerical Results}

Numerical results are obtained using MATHEMATICA 7 and are listed in Appendix B throughout the following points:

- $\quad$ Statistics of the apparent and absolute magnitudes of the F8V group

- Graphical representations:

a. Histograms for the apparent and absolute magnitudes of the stellar group

b. Graphical representations for the percentage errors for the mean and median

- $\quad$ Optimum choice of $\sigma$

- Distance analysis

- The frequency functions $\Phi(M)$ and $\Psi(m)$ of the absolute and apparent magnitudes for the F8V group

\section{CONCLUSION}

The aim of the present paper is three fold. First, to provide some basic descriptive statistics parameters for the apparent and absolute magnitudes of the nearby stars of spectral type F8V stars. Second, to establish the frequency functions $\Phi(M)$ and $\Psi(m)$ of the absolute and apparent magnitudes for these stars. Third, to compute the distance $r$ of these stars as a system assuming that they scatter around a mean absolute magnitude in a Gaussian distribution. The accuracy of the numerical results is satisfactory thus, the percentage error between $r$ and the mean value is less than $0.67 \%$. This percentage error indicates the applicability of the used method for distance determination in the ranges of the considered stellar group.

\section{REFERENCES}

[1] G. J. Habets, J. W. Heintze, "Empirical bolometric corrections for the main-sequence", Astronomy and Astrophysics, Vol. 46, pp. 193-237, 1981

[2] R. O. Gray, R. F. Garrison, "The Early F-Type Stars: Refined Classification, Confrontation with Strömgren Photometry, and the Effects of Rotation", Astrophysical Journal, Vol. 69, pp. 301-321, 1989

[3] H. I. Abdel-Rahman, I. A. Issa, M. A. Sharaf, M. I. Nouh, A. Bakry, A. I. Osman, A. S. Saad, F. Y. Kamal, Essam, "Statistical Gaussian Distribution Function as a Distance Indicator to Stellar Groups", Journal of Korean Astronomical Society, Vol. 42, No. 4, pp. 71-79, 2009

[4] M. A. Sharaf, I. A. Issa, A. Saad, "A method for the determination of cosmic distances", New Astronomy, Vol. 8, No. 1, pp. 15-21, 2003

[5] S. Cassisi, R. de Santis, A. M. Piersimoni, "The distance to Galactic globular clusters through RR Lyrae pulsational properties", Monthly Notices of the Royal Astronomical Society, Vol. 326, No. 1, pp. 342348, 2001

[6] B. W. Carney, B. C. Chaboyer, D. Dunca, T. Grand, D. W. Latham, A. Layden, A. Mcwilliam, A. Sarajedini, M. Shao, "Anchoring the Population II Distance Scale: Accurate Ages for Globular Clusters and Field Halo Stars", in American Astronomical Society Meeting Abstracts, Vol. 33, 2001

[7] E. D. Feigelson, G. J. Babu (eds), Statistical Challenges in Modern Astronomy, Springer-Verlag, 1992
[8] W. Gliese, H. Jahreiss, Preliminary Version of the Third Catalogue of Nearby Stars, VizieR Online Data Catalog, Vol. 5070, 1995

[9] ESA, The Hipparcos and Tycho Catalogues (ESA 1997), VizieR Online Data Catalog, Vol. 1239, 1997

[10] J. Kovalevsky, "Hipparcos and Tycho Catalogues," in Encyclopedia of Astronomy and Astrophysics, P. Murdin, Ed.: Encyclopedia of Astronomy and Astrophysics, 2000

[11] W. Gliese, Catalogue of Nearby Stars. Edition 1969, Veroeffentlichungen des Astronomischen Rechen-Instituts Heidelberg, Vol. 22, 1969

[12] W. Gliese, H. Jahreiss, "Nearby Star Data Published 1969-1978", Astronomy and Astrophysics, Vol. 38, pp.. 423-448, 1979

[13] R. van der Riet Woolley, Catalogue of stars within twenty-five parsecs of the Sun, Herstmonceux, Royal Greenwich Observatory, 1970

[14] D. Mihalas, J. Benney, Galactic Astronomy ,second edition. USA: Freeman and Company, 1981

[15] M. A. Sharaf, "Computations of the cosmic distance equation", Applied Mathematics and Computation, Vol. 174, No. 2, pp. 1269-1278, 2006

\section{APPENDIX}

\section{A. Appendix A: Input data}

TABLE I. DATA FOR SOME F8V NEARBY STARS

\begin{tabular}{|c|c|c|c|c|c|}
\hline Name & $\begin{array}{l}\text { Vmag } \\
\text { mag }\end{array}$ & $\begin{array}{l}\text { B-V } \\
\text { mag }\end{array}$ & $\begin{array}{l}\text { U-B } \\
\text { mag }\end{array}$ & $\begin{array}{l}\text { plx } \\
\text { mas }\end{array}$ & $\begin{array}{l}\text { Mv } \\
\text { mag }\end{array}$ \\
\hline Gl 34.1 & 5.07 & 0.51 & 0 & 55 & 3.77 \\
\hline Gl 41 & 4.82 & 0.53 & 0.12 & 76 & 4.22 \\
\hline Gl 55 & 4.96 & 0.58 & 0.1 & 82 & 4.53 \\
\hline Gl 61 & 4.09 & 0.54 & 0.06 & 57.4 & 2.88 \\
\hline NN 3109 & 5.52 & 0.53 & 0 & 53 & 4.14 \\
\hline NN 3115 & 5.79 & 0.55 & -0.01 & 49 & 4.24 \\
\hline G 81.3 & 6.1 & 0.48 & -0.04 & 40 & 4.11 \\
\hline Gl 87.1 & 5.67 & 0.56 & 0.07 & 34 & 3.33 \\
\hline NN 3161 & 6.02 & 0.54 & 0.04 & 44 & 4.24 \\
\hline NN 3175 & 5.78 & 0.52 & 0 & 45 & 4.05 \\
\hline Gl 115 & 8.17 & 0.55 & -0.01 & 61.9 & 7.13 \\
\hline Gl 128 & 5.06 & 0.57 & 0.12 & 53.3 & 3.69 \\
\hline Gl 147 & 4.28 & 0.57 & 0.08 & 59.4 & 3.15 \\
\hline Wo 9181 & 7.17 & 0.54 & 0.01 & 27 & 4.33 \\
\hline Gl 240.1 & 5.45 & 0.5 & -0.02 & 47.5 & 3.83 \\
\hline NN 3443 & 5.86 & 0.48 & -0.02 & 40 & 3.87 \\
\hline Gl 296.2 & 6.57 & 0.58 & 0.01 & 37 & 4.41 \\
\hline Gl 310.1 & 5.99 & 0.52 & 0.04 & 43.7 & 4.19 \\
\hline Gl 379.1 & 6.15 & 0.6 & 0.16 & 46.7 & 4.5 \\
\hline Gl 388.2 & 7.18 & 0.55 & -0.04 & 30 & 4.57 \\
\hline Gl 395 & 4.84 & 0.52 & -0.01 & 78 & 4.3 \\
\hline Gl 558.1 & 6.04 & 0.5 & -0.02 & 39 & 4 \\
\hline NN 3880 & 5.63 & 0.5 & 0 & 41 & 3.69 \\
\hline Gl 580.2 & 5.13 & 0.53 & 0.08 & 48.3 & 3.55 \\
\hline NN 3901 & 5 & 0.5 & 0.04 & 29.9 & 2.38 \\
\hline NN 3931 & 5.47 & 0.52 & 0.03 & 33.2 & 3.08 \\
\hline Gl 615.2 & 5.64 & 0.51 & 0 & 44.4 & 3.88 \\
\hline Gl 694.1 & 5.79 & 0.53 & 0.02 & 54.9 & 4.49 \\
\hline GI 743.1 & 4.87 & 0.52 & 0 & 68 & 4.03 \\
\hline Gl 768.1 & 5.11 & 0.55 & 0.07 & 59 & 3.96 \\
\hline Gl 773.4 & 5.66 & 0.49 & -0.02 & 51.6 & 4.22 \\
\hline Gl 794.2 & 5.12 & 0.53 & 0.01 & 55 & 3.82 \\
\hline Gl 811 & 5.7 & 0.5 & -0.04 & 45 & 3.97 \\
\hline Gl 827 & 4.22 & 0.49 & -0.12 & 115.5 & 4.53 \\
\hline Wo 9829 & 6.49 & 0.54 & -0.01 & 39 & 4.45 \\
\hline NN 4384 & 6.47 & 0.55 & -0.06 & 36 & 4.25 \\
\hline
\end{tabular}


TABLE II. Empirical RELATion BetweEn $\mathrm{M}_{\mathrm{v}}$ Versus $(B-V)_{0}$

\begin{tabular}{|c|c|c|c|}
\hline$(B-V)_{0}$ & $\mathrm{M}_{\mathrm{v}}$ & $(B-V)_{0}$ & $\mathrm{M}_{\mathrm{v}}$ \\
\hline-0.30 & -3.50 & 0.30 & 2.80 \\
\hline-0.25 & -2.30 & 0.40 & 3.35 \\
\hline-0.20 & -1.30 & 0.50 & 4.05 \\
\hline-0.15 & -0.50 & 0.60 & 4.60 \\
\hline-0.10 & 0.30 & 0.70 & 5.20 \\
\hline-0.05 & 0.90 & 0.80 & 5.70 \\
\hline 0.00 & 1.30 & 0.90 & 6.10 \\
\hline 0.05 & 1.55 & 1.00 & 6.60 \\
\hline 0.10 & 1.80 & 1.10 & 7.00 \\
\hline 0.20 & 2.25 & 1.20 & 7.45 \\
\hline & & 1.30 & 7.90 \\
\hline
\end{tabular}

TABLE III. THE $\mathrm{q}$ 'S COEFFICIENTS OF (7)

\begin{tabular}{|c|c|c|c|}
\hline $\mathbf{j}$ & $\mathbf{q}_{\mathbf{j}}$ & $\mathbf{j}$ & $\mathbf{q}_{\mathbf{j}}$ \\
\hline 1 & -0.786498 & 11 & 0.769777 \\
\hline 2 & 3.37819 & 12 & -0.421287 \\
\hline 3 & -1.96742 & 13 & 0.0908398 \\
\hline 4 & -10.6822 & 14 & 0.000500399 \\
\hline 5 & 20.8088 & 15 & -0.00573177 \\
\hline 6 & -11.6107 & 16 & 0.00172542 \\
\hline 7 & -5.11632 & 17 & -0.000284527 \\
\hline 8 & 10.3234 & 18 & 0.0000296064 \\
\hline 9 & -5.29694 & 19 & $-1.94463 \times 10^{-6}$ \\
\hline 10 & 0.467069 & 20 & $7.40906 \times 10^{-8}$ \\
\hline
\end{tabular}

\section{B. Appendix B: Output data}

B.1: Statistics of the apparent and absolute magnitudes of the F8V stars

- The average:

For Apparent Magnitudes $=5.63556$

For Absolute Magnitudes=4.04944

- The median (central value):

For Apparent Magnitudes $=5.65$

For Absolute Magnitudes $=4.08$

- The mode:

For Apparent Magnitudes $=5.79$

For Absolute Magnitudes $=\{3.69,4.22,4.24,4.53\}$

- The geometric mean:

For Apparent Magnitudes $=5.576$

For Absolute Magnitudes=3.9911

- The harmonic mean:

For Apparent Magnitudes $=5.51804$

For Absolute Magnitudes=3.93349

- The root mean square:

For Apparent Magnitudes $=5.69684$

For Absolute Magnitudes=4.1122

- The Quartiles:

For Apparent Magnitudes $=\{5.065,5.65,6.03\}$

For Absolute Magnitudes $=\{3.795,4.08,4.315\}$

- The range:

For Apparent Magnitudes $=4.08$

For Absolute Magnitudes $=4.75$

- The unbiased estimate of the variance:

For Apparent Magnitudes $=0.714385$

For Absolute Magnitudes $=0.526863$

- The maximum likelihood estimate of the variance:

For Apparent Magnitudes $=0.694541$

For Absolute Magnitudes $=0.512227$

- The unbiased estimate of the variance of the sample mean:

For Apparent Magnitudes $=0.019844$

For Absolute Magnitudes $=0.0146351$

- The unbiased estimate of the standard deviation: For Apparent Magnitudes $=0.845213$
For Absolute Magnitudes $=0.725853$

- The maximum likelihood estimate of the standard deviation :

For Apparent Magnitudes $=0.833391$

For Absolute Magnitudes $=0.715701$

- The unbiased estimate of the standard error(standard deviation)of the sample mean:

For Apparent Magnitudes $=0.140869$

For Absolute Magnitudes $=0.120975$

- The mean absolute deviation:

For Apparent Magnitudes $=0.620247$

For Absolute Magnitudes $=0.442253$

- The median absolute deviation:

For Apparent Magnitudes $=0.525$

For Absolute Magnitudes $=0.255$

- The interquartile range:

For Apparent Magnitudes $=0.965$

For Absolute Magnitudes $=0.52$

- The Quartiles:

For Apparent Magnitudes $=\{5.065,5.65,6.03\}$

For Absolute Magnitudes $=\{3.795,4.08,4.315\}$

- The coefficient of skewness:

For Apparent Magnitudes $=0.679003$

For Absolute Magnitudes=1.62311

- Pearson's first coefficient of skewness:

For Apparent Magnitudes $=-0.548185$

For Absolute Magnitudes $=\{1.48561,-0.704918,-0.787579,-1.98617\}$

- Pearson's second coefficient of skewness:

For Apparent Magnitudes $=-0.0512691$

For Absolute Magnitudes $=-0.126288$

- The quartile coefficient of skewness:

For Apparent Magnitudes=-0.212435

For Absolute Magnitudes $=-0.0961538$

- The kurtosis coefficient:

For Apparent Magnitudes $=4.00445$

For Absolute Magnitudes $=10.791$

- The kurtosis excess:

For Apparent Magnitudes $=1.00445$

For Absolute Magnitudes=7.7910

- The confidence interval of the population mean based on the normal distribution:

For Apparent Magnitudes $=\{5.35946,5.91165\}$

For Absolute Magnitudes $=\{3.81234,4.28655\}$

- The confidence interval for the population variance based on the chisquare distribution :

For Apparent Magnitudes $=\{0.469961,1.21557\}$

For Absolute Magnitudes $=\{0.346598,0.896487\}$

B.2 :Graphical Representations

The Histograms of the Apparent and Absolute Magnitudes of the stellar Group
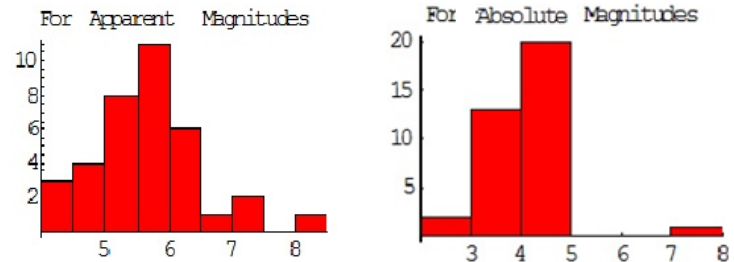

B.3: Optimum Choice of $\sigma$

Graphical representations of the percentage errors for the Mean and Median
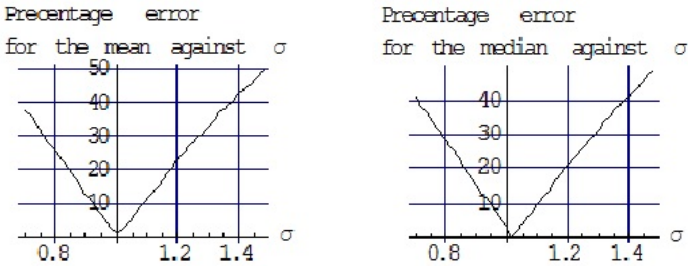
- The minimum value of the percentage error in the Mean is 0.553121 and occurs at $\sigma=1$.

- The minimum value of the percentage error in the Median is 0.18854 occurs at $\sigma=1.02$

- $\quad$ The optimum value of $\sigma$ is 1.01

\section{B.4: Distance Analysis}

- The value of $\mathrm{m}$ is 9.64125

- The value of $\bar{m}$ is 7.10681

- The value of $\sigma$ is 1.01

- The value of $\mathrm{M}_{0}$ is 5.45922

- The value of $\alpha$ is 2.50935

- The value of $\mathrm{y}$ is 2.49132

- The accuracy of the computed value of $y$ is $4.42657 \times 10$

- $\quad$ The distance $r$ of the stellar group as obtained from the statistical method is 21.5357 parsec

- The Median of the individual distances is 21.233 parsec

- The Mode of the individual distances is $\{18.1818,22.2222,25 ., 25.641\}$ parsec

- The geometric mean of the individual distances is 20.761 parsec

- The harmonic mean of the individual distances is 19.7835 parsec

- The mean value of the individual distances is 21.6796 parsec

- The percentage error between $r$ and $\hat{r}$ is $=0.663949$

B.5: Frequency functions of the absolute and apparent magnitudes for the F8Vstars

$$
\begin{aligned}
& \Phi(M)=0.398992 \times e^{-0.490148(-5.45922+M)_{2}} \\
& \Psi(m)=0.398992 \times e^{-0.490148(-7.12502+m)^{2}}
\end{aligned}
$$

